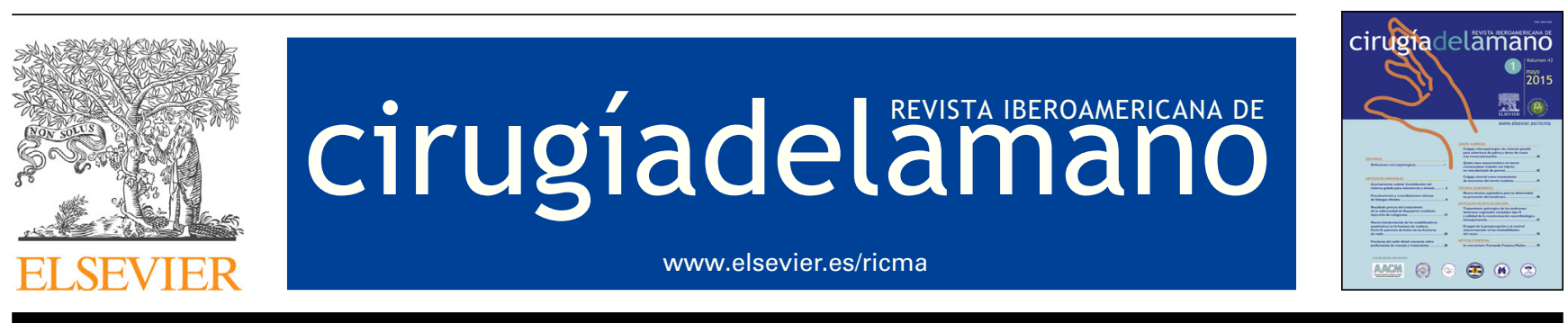

ARTÍCULO ORIGINAL

\title{
Osteotomía de acortamiento del hueso grande en la enfermedad de Kienböck asociada a varianza ulnar positiva o neutra
}

\author{
J.C. Sánchez Hernández*, M.C. Sanz Pascual y J.C. Gutiérrez Gómez
}

Servicio de Cirugía de la Mano y Nervio Periférico, Hospital Ramón y Cajal, Madrid, España

Recibido el 27 de junio de 2015; aceptado el 27 de septiembre de 2015

Disponible en Internet el 3 de octubre de 2015

\author{
PALABRAS CLAVE \\ Enfermedad de \\ Kienböck; \\ Osteotomía; \\ Acortamiento hueso \\ grande; \\ Varianza cubital \\ positiva
}

\begin{abstract}
Resumen
Objetivos: La enfermedad de Kienböck se asocia generalmente a cubitus minus y la descompresión quirúrgica más común es a nivel radial. El objetivo es conocer los efectos de realizarla en el hueso grande cuando se encuentra asociada a varianza cubital normal o positiva.

Material y métodos: Realizamos un estudio retrospectivo de los resultados de la osteotomía de acortamiento del hueso grande en la enfermedad de Kienböck sin cubitus minus. Hemos revisado a 6 pacientes, intervenidos entre 2006 y 2013 en el Hospital Universitario Ramón y Cajal (Madrid). El seguimiento medio fue 36 meses (12-93). Se registraron: escala de valoración del dolor (EVA), rango de movimiento de la muñeca, fuerza de prensión palmar y de la pinza, valoración funcional (DASH, Mayo Score), así como estudio radiológico y con RM.

Resultados: Se obtuvo una mejoría clínica y funcional de los parámetros estudiados, pero no de los radiológicos. En un caso la enfermedad evolucionó y el resto mantuvo los estadios radiológico (Lichtman) iniciales, consiguiendo la consolidación de la osteotomía en todos los casos. No se apreciaron complicaciones destacables.

Conclusión: La osteotomía de acortamiento del hueso grande es una técnica que oferta buenos y duraderos resultados en pacientes con enfermedad de Kienböck sin cubitus minus ni cambios degenerativos carpianos.

(c) 2015 SECMA. Publicado por Elsevier España, S.L.U. Este es un artículo Open Access bajo la licencia CC BY-NC-ND (http: // creativecommons.org/licenses/by-nc-nd/4.0/).
\end{abstract}

\footnotetext{
* Autor para correspondencia.

Correo electrónico: jcsanchezh@yahoo.es (J.C. Sánchez Hernández).
} 


\section{KEYWORDS}

Kienböck's disease;

Capitate shortening;

Osteotomy;

Ulnar positive

variance
Capitate shortening osteotomy in Kienböck's disease associated to variance ulnar positive or neutral

\begin{abstract}
Objectives: Kienbock's disease is usually associated with ulna minus and the most common surgical decompression is at radial level. The aim of this study was to evaluate the effects of the osteotomy of capitate in cases with normal or positive ulna variance.

Material and methods: We performed a retrospective study on 6 patients with Kienbock's disease without ulna minus who underwent capitate osteotomy between 2006 and 2013 at the Ramon y Cajal University Hospital. The mean time of follow-up after surgery was 36 months (12-93). We recorded: pain assessment scale (VAS), range of wrist motion, grip strength, pinch strength, functional and radiological evaluation.

Results: Clinical and functional improvement was obtained, except for radiological parameters. In one case the disease progressed and the rest remained the initial radiographic stages. Consolidation of the osteotomy was achieved in all cases. No remarkable complications were obtained.

Conclusion: Capitate shortening osteotomy, can be a safety good technique and offer lasting results, especially in Kienbock desease without ulnar minus or carpal degenerative changes.

(C) 2015 SECMA. Published by Elsevier España, S.L.U. This is an open access article under the CC BY-NC-ND license (http://creativecommons.org/licenses/by-nc-nd/4.0/).
\end{abstract}

\section{Introducción}

La etiología de la enfermedad de Kienböck (EK) es desconocida; se piensa que se debe a una pérdida de flujo sanguíneo en el hueso semilunar (Se), generalmente atribuida a la presencia de problemas circulatorios primarios. Es una patología muy restrictiva a veces, debido al dolor y a la limitación funcional de la mano que causa. La elevación en la presión intraósea por una mecánica carpiana alterada parece ser la vía más probable de esta necrosis avascular. Fue descrita en 1910 por Kienböck ${ }^{1}$ y clasificada inicialmente en 4 estadios radiológicos progresivos por Stahl ${ }^{2} \mathrm{y}$, posteriormente, Lichtman et al. ${ }^{3}$ subdividieron el estadio III, a su vez, en A, $B$ y $C$, en función de la presencia de colapso carpiano. Se han sugerido también clasificaciones basadas en la resonancia magnética $(\mathrm{RM})^{4,5}$ y en los hallazgos artroscópicos ${ }^{6}$ con implicaciones terapéuticas asociadas.

El curso natural de la enfermedad es impredecible, por lo que su tratamiento no está normalizado. Se han propuesto multitud de técnicas quirúrgicas para esta enfermedad en sus diferentes estadios. El objetivo del tratamiento sería disminuir las cargas de compresión sobre el semilunar para intentar conseguir así su revascularización ${ }^{7}$ y mejorar su pronóstico.

Se ha encontrado una relación estadísticamente significativa entre el cubitus minus y la enfermedad de kienböck, $\mathrm{y}$ es menos frecuente encontrarla asociada a una varianza cubital positiva o neutra ${ }^{8}$.

En estadios iniciales con varianza cubital negativa, el tratamiento conservador mediante inmovilización se ha propuesto con resultados insuficientes en muchos $\operatorname{casos}^{9,10}$. También se realizan en estos casos, para la disminución de las cargas sobre el Se, las osteotomías de acortamiento radial (OAR) tipo Nakamura o similar ${ }^{11,12}$, u osteotomías de alargamiento cubital $^{2}$, con los problemas de consolidación asociados a esta técnica ${ }^{13}$. En los estadios III B y III A de Lichtman, se han descrito artrodesis intercarpianas del hueso grande (HG) con el ganchoso ${ }^{14}$ o de la articulación escafo-trapecio-trapezoidea (STT) ${ }^{15,16}$, que si bien mejoran los resultados clínicos, su repercusión sobre las articulaciones radioescafoidea e intercarpianas están por determinar.

En pacientes con EK y varianza cubital positiva o neutra, el acortamiento radial o alargamiento cubital podrían producir una discrepancia de la articulación radio-cubital distal (ARCD) y un pinzamiento cubito-carpiano. En estos pacientes se proponen realizar las osteotomías del radio en cuña o como Almquist ${ }^{20,21}$ propuso, realizar la osteotomía de acortamiento en el HG (OAG) con/sin artrodesis HG-ganchoso, comunicándose varias series de pacientes operados con esta técnica quirúrgica (OAG), con buenos resultados clínicos y prometedores radiológicos ${ }^{22-24}$. Con la misma idea quirúrgica para descomprimir la fosa semilunar, se han publicado procedimientos en los que el acortamiento se consigue realizando una artrodesis capito-metacarpiana ${ }^{25}$. Incluso intentar descomprimir selectivamente el semilunar, mediante osteotomías de acortamiento parcial del HG, en las que se practica una osteotomía en $L$ de base distal, que acorta tan solo el lado ulnar del HG, para no afectar la columna carpiana radial ${ }^{26,27}$. Para mejorar la revascularización del semilunar, el aporte directo sanguíneo con injertos óseos vascularizados asociados o no a las anteriores técnicas, como los de radio distal, injerto de pisiforme, metacarpiano o injerto iliaco libre vascularizado, han sido comunicados $^{28-31}$.

En los estadios avanzados, con degeneración artrósica y colapso carpiano, se utilizan de forma paliativa las prótesis de semilunar ${ }^{17}$, con malos resultados a largo plazo $^{18}$, o bien las carpectomías proximales, artrodesis radiocarpianas o denervaciones totales de muñeca ${ }^{19}$.

El propósito de este estudio retrospectivo es evaluar los problemas técnicos de la OAG, sus eventuales complicaciones, su posible eficacia clínica en la evolución de la EK cuando se presenta asociada a una varianza cubital positiva 
o neutra, así como su acción sobre el hueso esponjoso del Se y en el resto del carpo.

\section{Material y método}

Realizamos un estudio retrospectivo que analiza los resultados obtenidos en el tratamiento quirúrgico de la EK asociada a varianza cubital positiva o neutra, cuando se ha realizado una OAG; con o sin otros gestos quirúrgicos añadidos.

Para ello se realiza un seguimiento de una serie de 6 pacientes (4 mujeres/ 2 varones), con una edad media de 46,6 años (38-57); 5 pacientes eran diestros y uno zurdo, y 4 presentaban su sintomatología en la mano dominante, operados por el Servicio de Cirugía de la Mano del Hospital Ramón y Cajal de Madrid entre los años 2006 y 2013. Todos ellos eran trabajadores manuales

Criterios de inclusión:

1. Muñecas con EK con varianza cubital neutra o positiva

2. Estadios iniciales de la EK, evaluados radiológicamente (radiografía $[R x]$ y $R M$ ) según la metodología de Lichtman:

3 casos en estadios de Lichtman II (esclerosis del semilunar sin colapso) y 3 Lichtman IIIA (colapso semilunar sin rotación escafoides) (figs. 1 y 2$)^{3}$.

3. Dolor y limitación de movilidad antiálgica en la muñeca durante la actividad.

4. Seguimiento durante más de un año (tiempo medio de seguimiento: 36 meses).

5. Pacientes con EK a los que se les había realizado una osteotomía de acortamiento del hueso grande (OAG), ya fuera sola o asociada a otros gestos quirúrgicos.

Técnica quirúrgica: bajo anestesia locorregional y con isquemia en la raíz del miembro superior, se practicó un abordaje cutáneo longitudinal dorsal medio, centrado sobre el HG previa visualización con fluoroscopia. Disección entre el tendón extensor común de los dedos y el extensor propio del índice, apertura del ligamento anular dorsal del carpo y capsulotomía, limitada proximalmente por la interlínea mediocarpiana a nivel del cuerno dorsal semilunar. El abordaje permitía la visualización del Se y la articulación escafo-semilunar.

Para preservar la vascularización del semilunar se llevó a cabo una mínima elevación de su cápsula dorsal. Si el semilunar se apreciaba notablemente fragmentado, con importante cambios degenerativos o pérdida de su cartílago articular, la osteotomía del HG estaba contraindicada.

Para poder realizar la osteotomía se realizó una disección capsular del segmento más distal del mismo, adyacente al ganchoso. El HG se expone perfectamente con ayuda de 3 separadores tipo Hoffman, que se mantienen delicadamente en las interlíneas articulares proximal y laterales al HG. Se realizó una osteotomía transversal oblicua, de dorsal a palmar y de proximal a distal, justo a nivel de la parte distal de la superficie articular proximal. Esta oblicuidad se realizaba para que el posterior material de síntesis fuese perpendicular al trazo de osteotomía y ejerciese un efecto de compresión del foco. El primer corte debía ser parcial y justo proximal a este, se realizaba otro corte paralelo, preservándose siempre la cápsula volar. El acortamiento usual era de
$3 \mathrm{~mm}$ de espesor (poco más que la anchura de la sierra). La cabeza del HG grande era un fragmento muy móvil. Por ello la osteotomía proximal se realizaba primero. Una vez realizada esta, la cabeza móvil se podía comprimir contra la parte distal del HG, usando el separador de Hoffman introducido en el espacio HG-Se, sin dañar el cartílago articular. En este momento, se pasaban una o 2 agujas de Kirschner paralelas de distal a proximal, a través de la osteotomía. A través de estas agujas se llevaba a cabo un brocado con el material apropiado canulado y finalmente se colocaban uno o 2 tornillos sin cabeza que aportan compresión al foco (fig. 3). En todo momento se realizaban controles sistemáticos radiológicos peroperatorios mediante fluoroscopia, para comprobar la correcta colocación del material de síntesis.

En los 3 primeros casos se realizó una denervación del nervio interóseo posterior, que en la actualidad consideramos innecesaria. En otro caso, se realizó previamente raspado de un quiste óseo en semilunar y relleno con injerto de esponjosa de extremidad distal de radio. Al comprobar quirúrgicamente el aspecto avascular del resto del $\mathrm{Se}$, se asoció la OAG.

Los pacientes se inmovilizaron mediante una férula de yeso palmar durante 2 semanas, hasta la retirada de la sutura cutánea; posteriormente se inmovilizaron con una muñequera limitadora de la flexo-extensión durante un mes. Se inició entonces el periodo rehabilitador, durante el primer mes cinesioterapia sin movilidad resistida y a partir de entonces ejercicios de fuerza.

Para la valoración del dolor se usó una escala visual analógica (EVA) en la que los pacientes respondían sobre la intensidad de su dolor ( 0 ausencia de dolor y 10 máximo dolor).

El rango de movilidad articular se midió mediante goniometría.

Las fuerza de prensión de puño y de pinza en 3 posiciones (PP, P-Lat. y $\mathrm{P}$ doble dedo) fueron medidas mediante un medidor Jamar en el preoperatorio, a los 6 meses de evolución (en algunos casos) y en la revisión final.

Para la valoración funcional de los pacientes se utilizó un cuestionario DASH.

El diagnóstico y la clasificación según estadios de Lichtman fueron realizados con exploración radiológica de las muñecas en proyecciones antero-posteriores y laterales (AP y L). Se confirmó mediante imágenes de RM, en las que aparecía una disminución marcada de la intensidad de la señal con las secuencias T1 (fig. 2).

En la revisión final de la serie de casos, efectuada entre enero y marzo del 2015, se realizó una evaluación funcional de la muñeca según la escala de Cooney (MAYO modificada $)^{35}$. En esta se asocia un control de la movilidad de la muñeca operada, evaluación del dolor, retorno a la actividad y fuerza de la garra medida mediante un medidor de Jamar. Estas medidas se compararon con el lado contralateral.

También se aplicó una escala de valoración subjetiva de satisfacción con el resultado de su cirugía, mediante una escala del 1 (insatisfacción con intenso dolor e importante limitación funcional) al 5 (satisfacción completa sin dolor ni limitación funcional).

Se registró también el tiempo de reincorporación a su trabajo habitual después de la cirugía. Se realizaron radiografías de control, en proyecciones AP y L para apreciar la 

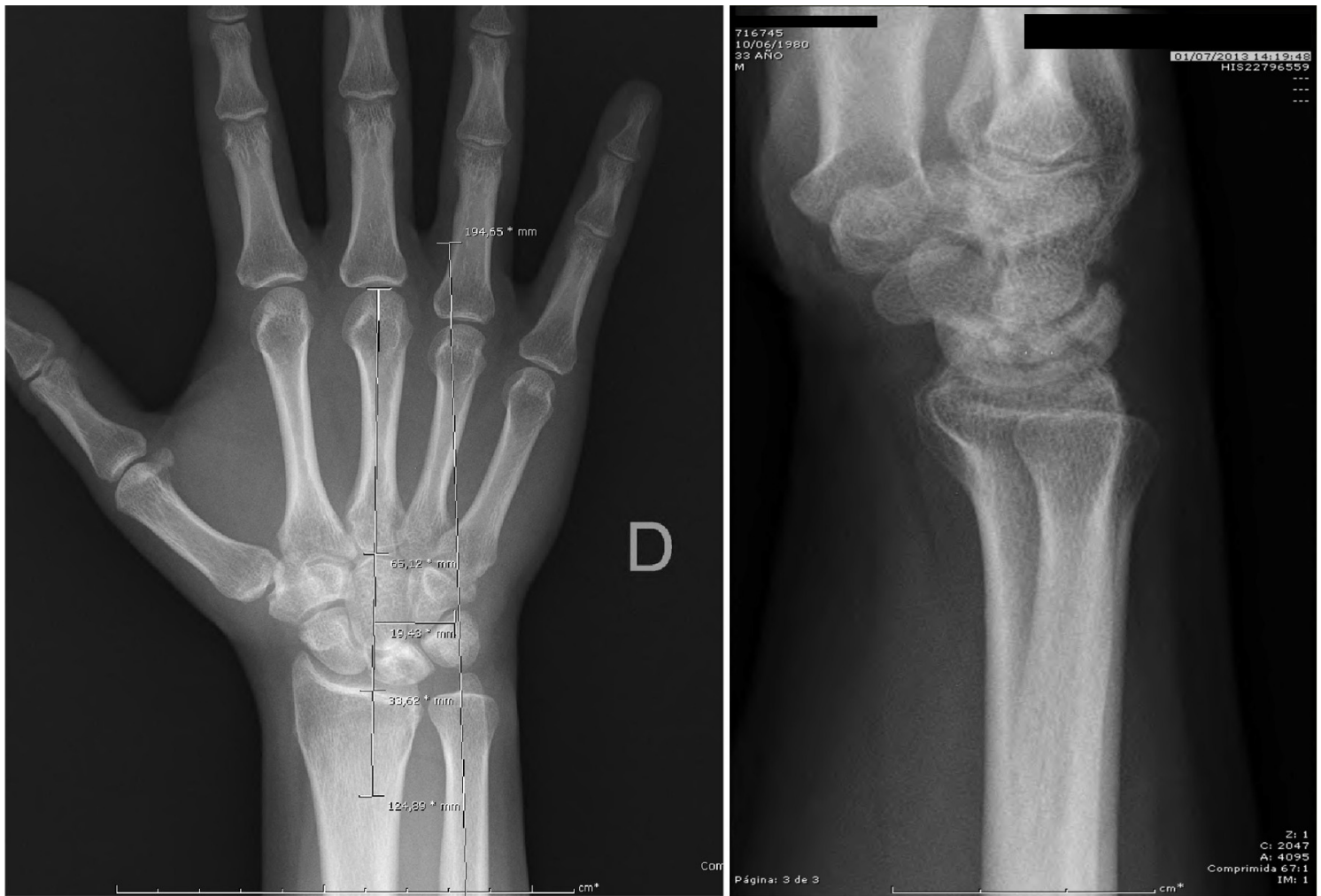

Figura 1 Rx simple AP y lateral en la que se aprecia el proceso de alteración estructural en el hueso semilunar, a la vez que una varianza cubital positiva/neutra.

evolución radiológica de la enfermedad, la estabilidad del material de síntesis en su caso y el aspecto de la trama ósea del HG, y una revaluación del estadio según la clasificación de Lichtman. Se midieron tanto la relación entre la altura del carpo y la longitud del 3. ${ }^{\mathrm{er}}$ metacarpiano (índice de Youm), como los tamaños radiológicos carpianos y tipos de semilunar según Antuña Zapico. Se realizó también una exploración de las muñecas con RM. Las secuencias coronales T1 y T2, «spir» y sagitales T1 con inyección de gadolinio pueden permitir precisar el impacto de la osteotomía OAG sobre el Se, el HG y las articulaciones intercarpianas en comparación con las imágenes prequirúrgicas previas.
La reincorporación a su trabajo habitual se decidió en función de la evolución clínica y radiológica. Se realizan revisiones en consulta externa a los 15, 45 días y posteriormente a los 6 meses y anualmente. Y, por último, la valoración final de la serie en la actualidad. El seguimiento medio de revisión de la serie fue de 36 meses (entre 12 y 93 meses).

\section{Resultados}

El tiempo quirúrgico medio fue de $45 \min$ (40- $54 \mathrm{~min}$ ).

La evaluación del dolor con arreglo a una escala visual analógica (EVA) preoperatoria obtuvo una puntuación media

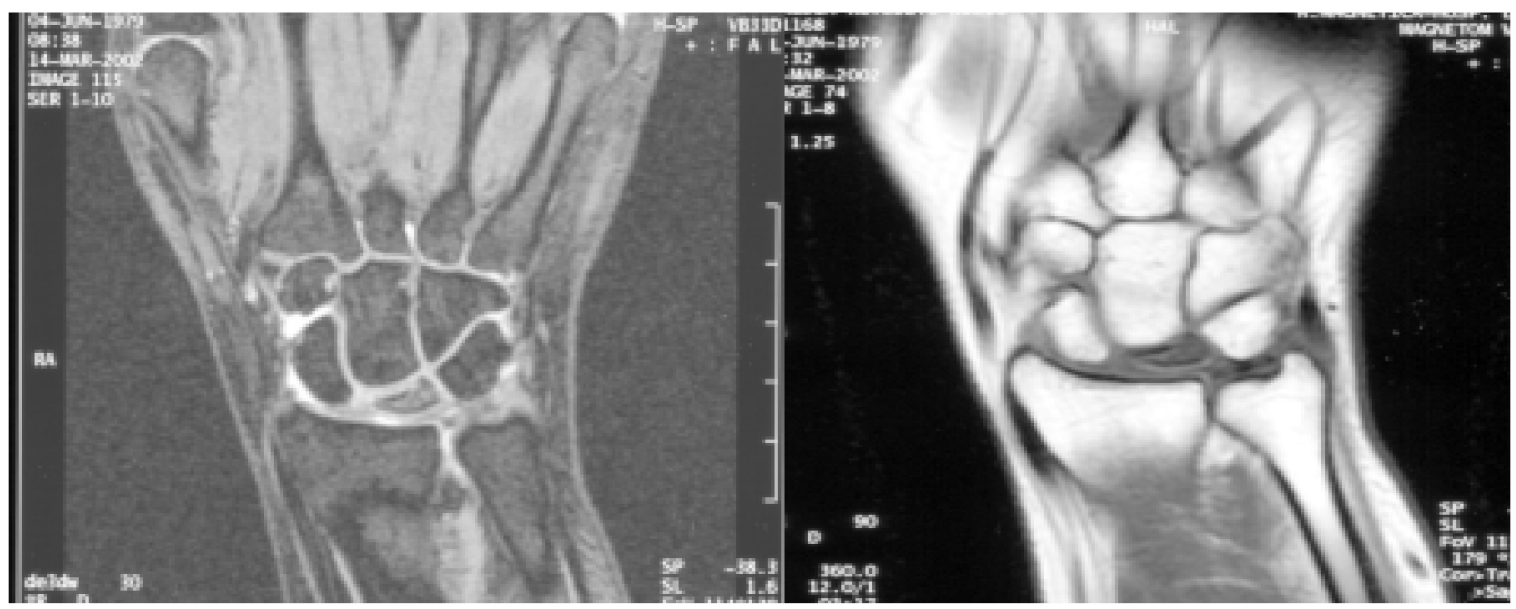

Figura 2 RM: alteración en la intensidad de la señal del hueso semilunar. Varianza cubital neutra. 


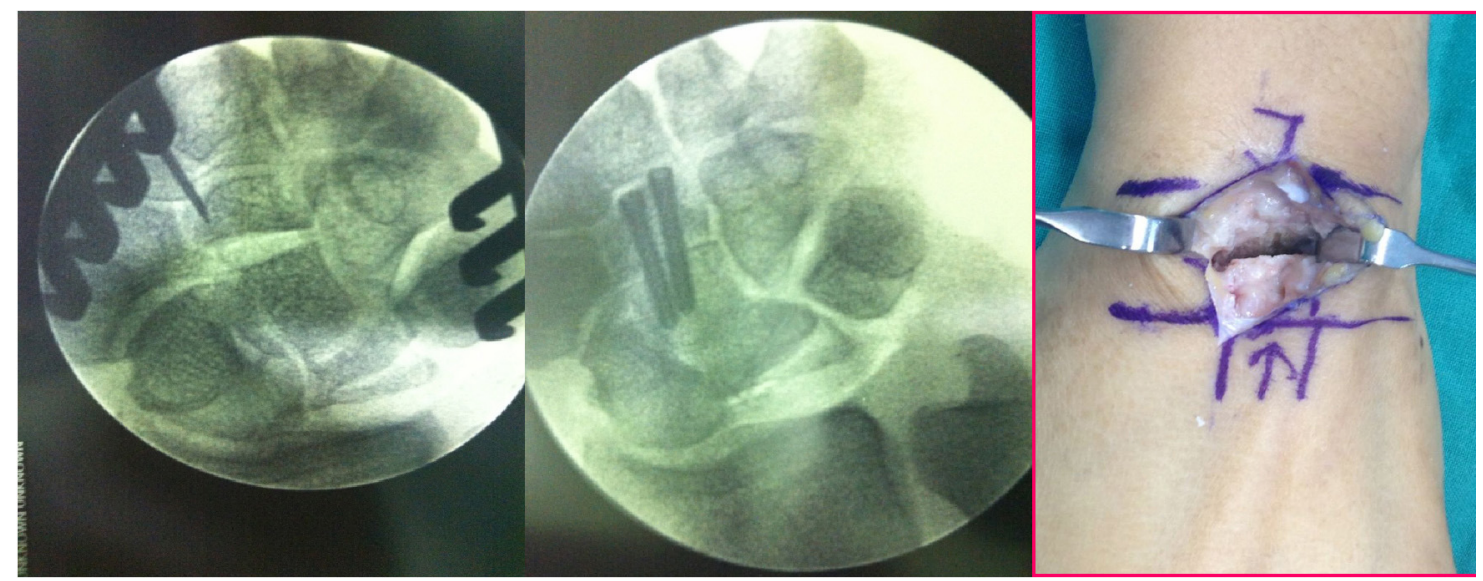

Figura 3 Imagen intraoperatoria de osteotomía transversa de hueso grande y síntesis de la misma con 2 tornillos canulados a compresión.

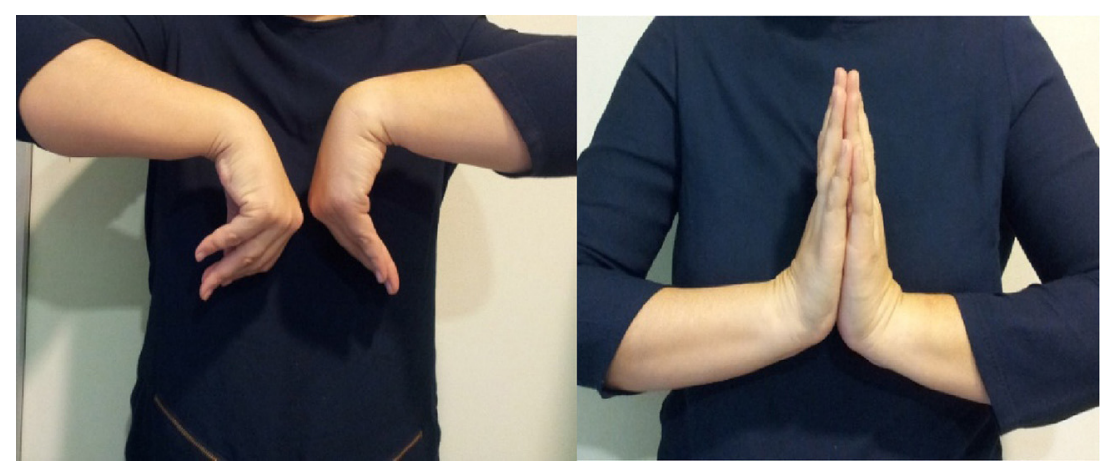

Figura 4 Evaluación de movilidad articular a los 2 meses postoperatorios.

de 7,9 (7-9) y en la revisión final una puntuación media de $1,7(0,5-4)$, con una puntuación de la muñeca contralateral de 1,1 .

El rango de movimiento de la muñeca medio mejoró en todos los casos con respecto a los valores preoperatorios, registrándose unos valores prácticamente similares al de la muñeca contralateral en casi todos los casos (figs. 4 y 8), si bien en algún caso se observó en los primeros meses una limitación para la flexión palmar de la muñeca. Los datos de movilidad preoperatoria y postoperatoria se muestran en la tabla 1.

Se obtuvo una mejoría apreciable en la potencia tanto de la garra como de la fuerza de la pinza en sus 3 posiciones en todos los casos (fig. 5). Comparando con los valores medios medidos en la mano contralateral, se obtuvieron medidas significativamente mejores después del tratamiento quirúrgico, registrándose en los valores prequirúrgicos una disminución de fuerza de un 38,20\% y en la revisión final la fuerza registrada era del $85,4 \%$, con respecto al lado contralateral.

La puntuación media en la escala DASH en la revisión realizada fue de $10,09(8,82-11,36)$. La puntuación media con arreglo a la escala de Cooney fue de 82,5 (tabla 2).

Los pacientes puntuaron su grado de satisfacción preoperatoria y postoperatoria, siendo la puntuación media preoperatoria de $2(0-3)$ y a los 6 meses postoperatorios de $4(3-5)$.
Todos los pacientes de la serie retornaron a sus trabajos habituales (actividades laborales manuales en todos los casos) en un tiempo medio de 4 meses (3-7 meses en el caso de un carnicero que también refería modificación en algunas de sus tareas profesionales).

No se apreciaron modificaciones en el índice de Youm después de la cirugía, obteniéndose un valor medio de $0,47 \mathrm{~mm}$. Cuatro semilunares presentaban un tipo ॥ (fig. 1) y 2 un tipo III según la clasificación morfológica de Antuña Zapico. Tampoco hubo cambios significativos en las mediciones radiológicas del semilunar antes y después de la cirugía. En todos los pacientes se consiguió la consolidación del foco de la osteotomía a los 3,5 meses de media (3-5 meses) y en ningún caso se constató una necrosis del polo proximal del HG.

En 4 de los pacientes revisados se realizó RM al año de evolución tras la cirugía, apreciándose en un caso la progre-

Tabla 1

\begin{tabular}{lll}
\hline Movilidad & Preoperatorio & Postoperatorio \\
\hline Flexión & $30^{\circ}\left(10-50^{\circ}\right)$ & $45^{\circ}\left(30-60^{\circ}\right)$ \\
Extensión & $42,3^{\circ}\left(32-60^{\circ}\right)$ & $60^{\circ}\left(60-75^{\circ}\right)$ \\
Incl. radial & $10^{\circ}$ & $11,5^{\circ}$ \\
Incl. cubital & $26,5^{\circ}$ & $25^{\circ}$ \\
\hline
\end{tabular}




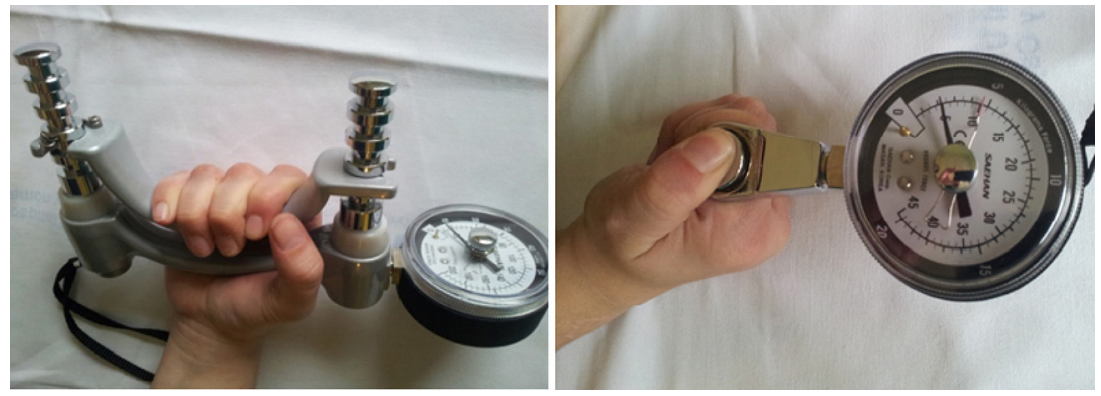

Figura 5 Evaluación de la Fuerza de la Garra y la pinza.

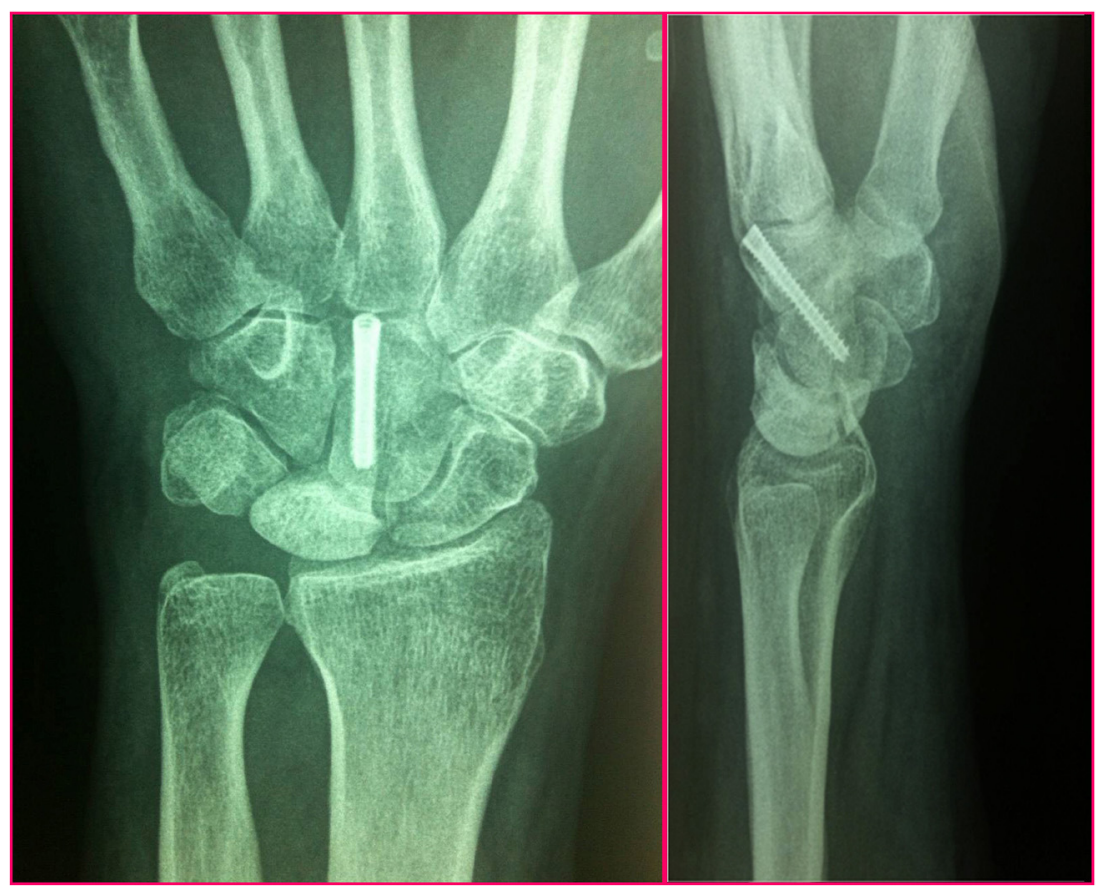

Figura 6 Control radiológico postoperatorios.

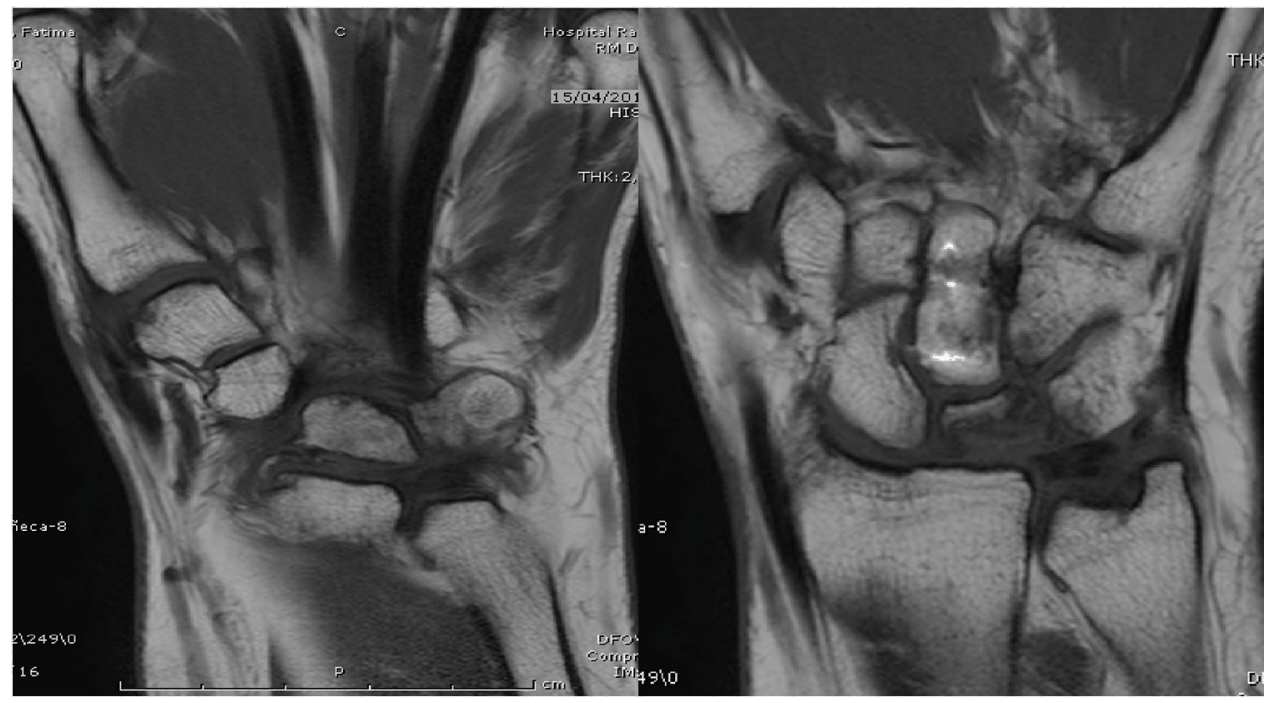

Figura 7 RM al año de la Osteotomía de Acortamiento del Hueso Grande. 


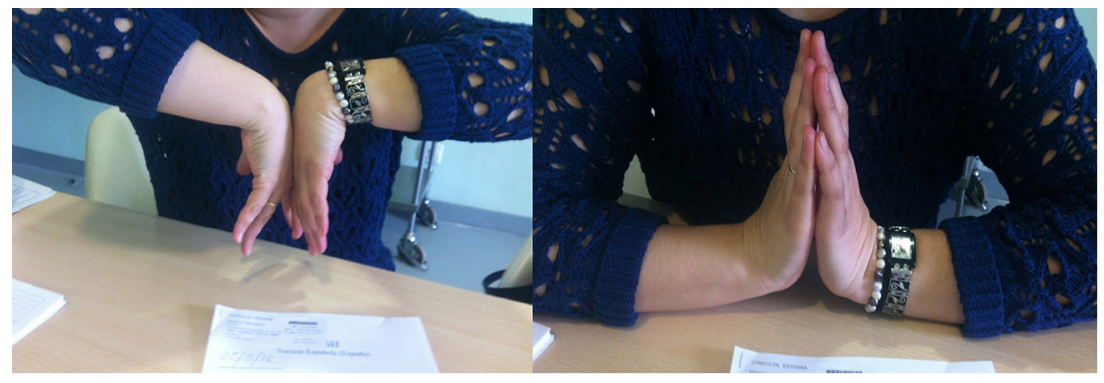

Figura 8 Movilidad de muñeca después de un año.

Tabla 2 Escala de Cooney (MAYO modificada)

\begin{tabular}{|c|c|c|c|}
\hline & & \multicolumn{2}{|c|}{ Puntos } \\
\hline \multirow[t]{4}{*}{ Dolor } & (25puntos) & Ausente & 25 \\
\hline & & Ocasional & 20 \\
\hline & & Moderado, tolerable & 15 \\
\hline & & Severo, Intolerable & 0 \\
\hline \multirow[t]{4}{*}{ Funcionalidad } & (25 puntos) & Igual a previa & 25 \\
\hline & & Adaptación de puesto & 20 \\
\hline & & Cambio de trabajo & 15 \\
\hline & & Imposibilidad trabajo & 0 \\
\hline \multirow[t]{4}{*}{ Movilidad } & (25 puntos) & $120^{\circ}$ de Flexo-extensión & 25 \\
\hline & & $90^{\circ}-120^{\circ} \mathrm{F}-\mathrm{Ext}$ & 15 \\
\hline & & $30^{\circ}$ a $60^{\circ} \mathrm{F}$-Ext & 10 \\
\hline & & $30^{\circ}$ o menos F-Ext & 5 \\
\hline \multirow[t]{9}{*}{ Fuerza } & (25 puntos) & $100 \%$ del lado contralateral & 25 \\
\hline & & 75 a $100 \%$ & 15 \\
\hline & & 50 a $75 \%$ & 10 \\
\hline & & 25 a $50 \%$ & 5 \\
\hline & & 0 a $25 \%$ & 0 \\
\hline & \multirow[t]{4}{*}{ Resultado } & Excelente & $90-100$ puntos \\
\hline & & Bueno & 80 a 90 puntos \\
\hline & & Medio & 65 a 80 puntos \\
\hline & & Malo & menos de 65 puntos \\
\hline
\end{tabular}

sión de la EK de un estadio ॥A de Lichtman a un estadio ॥ con agravamiento de la fragmentación del Se. No se detectaron aflojamientos del material de síntesis. No se encontraron diferencias reseñables en las RM realizadas en el estudio final actual con respecto a las anteriores.

En 2 de los casos, aparte de cambios posquirúrgicos, esclerosis y edema parcheado en semilunar, se apreciaron discretos signos de revascularización (fig. 7), si bien no encontramos en ningún caso signos de regresión o mejora del estadio inicial.

En cuanto a las complicaciones, uno de los pacientes presentó limitación de su balance articular con disminución de la flexión palmar $\left(30^{\circ}\right)$. En ese mismo caso se apreció al año de evolución la progresión de la enfermedad, con fragmentación del semilunar, si bien sin mucha rotación del escafoides.

También en 2 casos se apreció una tendencia a formar un osteofito dorsal del semilunar, con ligera desviación dorsal del mismo.

No existieron complicaciones de partes blandas ni ningún caso de seudoartrosis en el foco de osteotomía u osteonecrosis proximal del HG (fig. 6). No se evidenciaron complicaciones intercarpianas ni de síndrome doloroso regional complejo tras la cirugía. En un caso se necesitó la retirada del material de osteosíntesis por molestias locales con la entrada del tornillo.

\section{Discusión}

La EK es de etiología desconocida y su evolución sin tratamiento no siempre es mala, ya que la artrosis de muñeca tras una necrosis avascular del semilunar no se da en todos los $\operatorname{casos}^{32}$. Si a esto unimos que para evaluar la eficacia de una determinada técnica quirúrgica y su efecto, modificador o no, en la evolución natural de la EK es necesario un seguimiento largo de los casos, como afirman Axelsson y Moberg (Le Poignet, 1983) de unos 10 años, y que además las series de pacientes que presentan EK asociada a varianza cubital positiva o neutra son siempre muy cortas y su presentación en el tiempo esporádica, se hace muy difícil sacar conclusiones que sean estadísticamente significativas. 
Existen diferentes alternativas de actuación frente a una EK, desde el meramente conservador hasta múltiples técnicas quirúrgicas, propuestas sobre todo cuando los síntomas lo aconsejan. Las artrodesis intercarpianas (STT O HGganchoso) en estadios iniciales y en los más avanzados (III BC) cuando el escafoides se horizontaliza, buscando recolocar el escafoides correctamente y descomprimir el semilunar ${ }^{3-14}$ limitan bastante la movilidad de la muñeca ${ }^{12-33}$. Las OAR son las más comúnmente utilizadas, pero en pacientes con varianza cubital positiva pueden ocasionar una disociación radio cubital o un «impingement» ulno-carpiano, por el relativo alargamiento cubital secundario. Por esto se realizan las osteotomías radiales balanceadas en cuña, intentando un efecto de pivote sobre la carilla radial de la ARCD.

El acortamiento del radio, cuando este no es más largo que el cúbito, podría no corregir la supuesta causa de la enfermedad, aparte de los conflictos mencionados sobre el espacio cúbito-carpiano y la ARCD ${ }^{11}$, si bien algunas series reportadas no los encuentran ${ }^{12}$. Por esto, en estos casos, Almquist propuso realizar la OAG, asociada a una artrodesis HG-ganchoso. En nuestros casos no se realizó la artrodesis HG-G, si bien sí se realizaron otros gestos quirúrgicos (denervaciones o relleno de quiste del Se) que, como en los casos de Almquist, dificultan analizar el papel aislado de la OAG. Por sí sola esta artrodesis no parece actuar disminuyendo las fuerzas de presión en la fosa del semilunar, como demuestran los estudios experimentales de Trumble T $^{37}$ y es tan solo cuando se utiliza asociada a la OAG cuando disminuye la presión en dicha fosa, no evitando que las fuerzas se trasladen también a la vertiente radio-escafoidea. Por otra parte, este gesto alarga los tiempos quirúrgi$\cos \sin$ aportar grandes beneficios como apuntan otros autores $^{22}$.

La descompresión de la fosa semilunar radial ha sido objeto de muchos estudios experimentales. La OAG disminuye las cargas del semilunar en un $66 \%$, pero a costa de incrementar las cargas un $150 \%$ sobre la articulación trapecio-escafoidea como concluyen Horii et al. ${ }^{34}$ y también Weber et al. ${ }^{35}$. La OAR con horizontalización propuesta por Nakamura y comunicados sus resultados a largo plazo por Watanabe et al. ${ }^{36}$ disminuye un $23 \%$ la presión en la fosa semilunar.

La cabeza del HG recibe una considerable vascularización a través de la cápsula volar, penetrando justo por el margen articular. Es importante realizar la osteotomía en la zona distal del HG, respetando la vascularización en la cabeza. La principal complicación y contraindicación descrita de esta técnica es la necrosis avascular del polo proximal del hueso. En nuestra serie, no tuvimos ningún caso de seudoartrosis o de osteonecrosis de este fragmento, quizás porque la vascularización palmar aportada por los pedículos palmares de la arcada intercarpiana y las ramas de la arcada palmar profunda, así como el abordaje dorsal, realizado con una capsulotomía mínima y cuidadosa, explicarían la ausencia de estas complicaciones ${ }^{22}$. En el seguimiento posquirúrgico no se registraron complicaciones de importancia, salvo las reflejadas, consiguiéndose la consolidación de la osteotomía en todos los casos.

Todos los pacientes operados presentaban sintomatología dolorosa, limitación funcional preoperatoria e integridad estructural de semilunar y por su varianza cubital elegimos la OAG frente a la de acortamiento radial para su descompresión.

Para intentar minimizar el efecto del acortamiento del HG sobre el carpo radial, la resección ósea de la osteotomía no debe ser muy grande, con $3 \mathrm{~mm}$ es suficiente para conseguir disminuir la presión en la fosa lunar sin afectar en exceso a la dinámica carpiana restante. Se han descrito otras técnicas quirúrgicas que intentan corregir este efecto, como la osteotomía del hueso grande parcial en «L» descrita por Moritomo et al. ${ }^{26}$, si bien por su dificultad técnica y posible desarrollo de artrosis por el escalón articular de la cabeza del HG nos decantamos por la técnica reportada. Waitayawinyu et al. ${ }^{30}$ proponen asociar a la OAG un injerto óseo vascularizado de la base del $2 .^{\circ}$ o el $3 .^{\circ}$ metacarpianos con buenos resultados clínicos.

Asegurar que una técnica quirúrgica hace que el Semilunar recupere su aporte vascular normal y regenere su estructura osea es difícil de evaluar. La Osteotomía de Acortamiento del Hueso Grande podría tener algún efecto sobre esta recuperación. Si bien, a tenor de los pocos casos presentados en este estudio y de la asociación en varios de ellos de otros gestos quirúrgicos, no es fácil saber en que medida la OAG por si sola, influye en el resultado final. Si bien es el gesto quirúrgico común realizado en todos los casos de la serie. Una exploración postoperatoria mediante Rx, TAC y RM puede ser de gran utilidad. En 4 de los 6 pacientes en la RM se apreciaba algún grado de revascularización en el semilunar, sobre todo en la parte dorsal del mismo, donde con frecuencia se detectó una neoformación ósea en el cuerno dorsal (osteofito dorsal). En ningún caso se detectó mejoría en los estadios previos de la enfermedad ni normalización de la trama ósea del semilunar. Por el contrario, en las RM realizadas no se detectaron cambios degenerativos destacables a nivel de la STT o de la vertiente radial del carpo.

No hay una técnica quirúrgica idónea para todos los casos de la EK; dependerá de la varianza cubital, la importancia de la sintomatología, el grado evolutivo o estadio del proceso y factores como la profesión, afecciones o edad del paciente.

\section{Conclusión}

La OAG, fijada con tornillos por vía dorsal del carpo, para el tratamiento de la EK no asociada a cubitus minus ni colapso carpiano podría ser una técnica a utilizar, con pocas complicaciones y que oferta buenos y duraderos resultados clínicamente, sobre todo en pacientes sin muchos cambios degenerativos carpianos.

Si bien no es posible concluir que disminuya el porcentaje de la progresión de la enfermedad, parece incrementar la fuerza de la muñeca, el grado de satisfacción de los pacientes y los resultados funcionales con respecto a los previos.

Esta técnica tampoco impediría la realización de cualquier otra cirugía adicional, como osteotomía radial, carpectomía proximal, artrodesis intercarpianas o radiocarpianas, si la progresión sintomática de la enfermedad lo hiciera necesario.

\section{Conflicto de intereses}

Los autores declaran no tener ningún conflicto de intereses. 


\section{Agradecimientos}

A todos los revisores del artículo, por sus comentarios y recomendaciones.

\section{Bibliografía}

1. Kienböck R. Uber traumatiche Malazie des mondbeis und ihre Flgenzustande: Entartungsformen unkompresions fracturen. Fortsch Geb Roentngen. 1910-1911;16:77-103.

2. Stahl F. On Lunatomalacia (Kienböck's disease). Acta Chirurg Scand. 1947;1 Supl 126.

3. Lichtman D, Lesley N, Simmons S. The classification and treatment of Kienböck disease: The state of the art and a look at the future. J Hand Surg. 2010;35:549-54.

4. Lamas C. La enfermedad de Kienbock. Barcelona: J.M. Bosch Editor; 2005.

5. Schuind F, Eslami S, Ledoux P. Kienböck's desease. J Bone Joint Surg. 2008:133-9, 90A: .

6. Bain GL, Begg M. Artroscopic assessment and classification of Kienbock's disease. Tech Hand Up Extrem Surg. 2006;10(1):8-13.

7. Allan C, Joshi A, Lichtman DM. Kienböck's disease: Diagnosis and treatment. J Am Acad Orthop Surg. 2001;9:128-36.

8. Hulten $O$. Uber anastomische variationen den Handgelenkknochen. Acta Radiol Sacand. 1928;9:255-68.

9. Milkelsen SS, Gelinek J. Poor function after non operative treatment of Kienbockis disease. Acta Orthop Scand. 1987;58:241-3.

10. Salmon J, Stanley JK, Trail A. Kienböck's disease. Conservative treatment versus radial shortening. J Bone Joint Surg Br. 2000;82:820-3.

11. Nakamura R, Imaeda T, Miura T. Radial Shortening for Kienböck's disease factors affecting the operative result. J Hand Surg Am. 1990;15B:40-4.

12. Botelheiro JC, Silverio S. Tratamento da doenca de Kiembock com colapsocárpiano (Lichtman IIIb) com osteotomia de encurtamento do radio -16 casos. Rev Iberamer Cir Mano. 2014;42(2):139-43.

13. Trail IA, Linscheid RL, Quenzer DE, Scherer PA. Ulnar lengthening and radial recession procedures for Kienbock's disease -long-term clinical and radiographic follow-up. J Hand Surg Br. 1996;21:169-76.

14. Inoue G. Capitate-hamate fusion for Kienbock's disease: Good results in 8 cas for 3 years. Acta Orthop Scand. 1992;63:560-2.

15. Meier RA, van Griensven M, Krimer H. Scaphotrapezoid (STT)-arthrodesis in Kienböck's disease. J Hand Surg. 2004;29(6):580-4.

16. Voche P, Bour C, Merie M. Scapho-trapezio-trapezoid arthrodesis in treatment of Kienbock's disease a study of 16 cases. J Hand Surg Br. 1992; 17:5-11.

17. O'Flanagan SJ, Curtin J. Lunate Slastic arthropasty in Kienbock's disease. J R Coll Surg Edin. 1992;37:52-6.

18. Viljakka T, Tallroth K, Vastamiaki M. Long- term outcome (22-36 years) of silicone lunate arthroplasty for Kienböck's disease. J Hand Surg Eur. 2014;39E(4):405-15.

19. Foucher G, da Silva JB, Ferreres A. La denervation totale du poignet. A propos de 50 cas. Rev Chir Orthop. 1992;78: 186-90.
20. Almquist EE. Capitate shortenig with capitate-hamate fusion. En: Watson, HK, editor. The Wrist. Philadelphia: LippincotWilliams Wilkins; 2001. p. 785-792.

21. Almquist EE. Capitate Shortening in the treatment of Kienböck's disease. Hand Cliniccs. 1993;9:505-12.

22. Rabarin F, et al. Capitate osteotomy in Kienbóck's diesase in twelve cases. Clinical an radiological results at five years followup. Chir Main. 2010;29(2):67-71.

23. Afshar $A$, et al. Lunate revascularization after capitate shortening osteotomy in Kienböck's disease. J Hand Surg. 2010;35A:1943-6.

24. Gay A, Parrate S, Glard Y, Legre R. Isolated capitate shortening osteotomy for early stage of Kienböck's disease with neutral ulnar variance. Plast Reconstr Surg. 2009;124: $560-6$.

25. Fouley E-H, Sadeck AF, Amin MF. Distal capitate shortening with capitometacarpal fusion for manegement of the early stages of Kienbock's disease with neutral ulnar variance: Case series. J Orthop Surg Rev. 2014;9(1):86.

26. Moritomo H, Murase T, Yoshikawa H. Operative technique of a new descompression procedure for Kienböck's disease: Partial capitate shortening. Tech Hand Upper Extrem Surg. 2004;8(2):110-5.

27. Citlak A, Akgun U, Bukut T, Tahta M, Dirim Mete B, Sener M, et al. Partial capitate shortening for Kienbock's disease. J Hand Surg Eur. 2014, pii1753193414562355.

28. Sunagawa T, Bishop AT, Muramatsu K. Vascularised pedicle grafts from the dorsal distal radius design and application for carpal pathology. En: Saffar P, Amedio PC, Foucher G, editores. Current practice in hand surgery. London: Martin Dunitz Ltd; 1997. p. 307-313.

29. Bochud RC, Bucher U. Kienbock's disease, early stage 3 -height reconstruction an core vascularization of the lunate. J Hand Surg Br. 1994;19:466-77.

30. Waitayawinyu T, Chin S, Luria S, Trumble T. Capitate shortening osteotomy with vascularized bone grafting for the treatment of kienböck's disease in th ulnar positive wrist. J Hand Surg. 2008;33A:1267-73.

31. Fujiwara H, Oda R, Morisaki S, Ikoma K, Kubo T. Long-term results of vascularized bone graft for stage III Kienbock's disease. J Hand Surg Am. 2013;38(5):904-8.

32. Ramunssen F, Schantz K. Radiological aspects of lunatomalacia. Eur J Radio. 1987;7:199-202.

33. Condit D, Idler R, Fischer T, Hastings H. Preoperative factors and outcome after lunate descompression for Kienbock's disease. J Hand Surg Am. 1994;18:691-6.

34. Horii E, Garcia Elias M, Kn AN, Bishop AT. Effect on force transmission across the carpus in procedures used to treat Kienbóck's Disase. J Hand Surg Am. 1990;15:393-400.

35. Weber KD, Schmelz R, Peimer CA, Wagenpfeil S, Machens HG, Lohmeyer JA. Biomechanical effect of isolated capitate shortening in Kienbock's disease: An anatomical study. J Hand Surg Eur. 2013;38:500-7.

36. Watanabe T, Takahara M, Tsuchida H, Yamahara S, Kikuchi N, Ogino T. Long-term floor-up of radial shortening osteotomy for Kienböck's disease. J Bone Joint Surg (Am). 2008;90:1705-11.

37. Trumble T, Glisson R, Searer A, Urbaniak J. A biomechanical comparison of methods for treating Kienböckś disease. J Hand Surg. 1996;11A:88-93. 